\title{
An unexpected route for otolaryngology bacterial contamination with a Venturi atomizer*
}

\author{
Te-Ming Tseng ${ }^{1}$, Po-Yueh Chen ${ }^{2,3}$, How Tseng ${ }^{4}$, Hsiu-Chen Lin' ${ }^{5,6}$, \\ Ching-Yu Chang ${ }^{5}$, Shih-Han Hung 1,3,4 \\ 1 Department of Otolaryngology, Taipei Medical University Hospital, Taipei, Taiwan \\ 2 Department of Otolaryngology Head \& Neck Surgery, Taipei Medical University-Shuang Ho Hospital, Taipei, Taiwan \\ ${ }^{3}$ Department of Otolaryngology, School of Medicine, Taipei Medical University, Taipei, Taiwan \\ ${ }^{4}$ Graduate Institute of Medical Sciences, Taipei Medical University, Taipei, Taiwan \\ ${ }^{5}$ Department of Laboratory Medicine, Taipei Medical University Hospital, Taipei, Taiwan \\ ${ }^{6}$ Department of Pediatrics, College of Medicine, School of Medicine, Taipei Medical University, Taiwan
}

Rhinology 52: 156-161, 2014

DOI:10.4193/Rhino13.124

*Received for publication:

August 12, 2013

Accepted: November 6, 2013

\begin{abstract}
Background: The Venturi-principle atomizer is a commonly used device in otolaryngology practices. The purpose of this study is to evaluate the possible route of bacterial contamination from the nasal vestibule to the atomizer tip through the jet airflow created during the use of the Venturi atomizer.
\end{abstract}

Methods: Thirty nostrils from 15 enrolled volunteers were tested. The aerosols generated by spraying sterilized saline into the nostrils were collected using a specially made aerosol-collecting nozzle cap. The collected samples were sent for bacterial culture, and nasal vestibular swab cultures were performed for comparison.

Results: In the aerosol-exposed group, 18 out of 30 samples (60\%) were positive for bacterial growth, confirming the bacterial contamination from the nasal vestibule to the atomizer tip through the reverse jet airflow. The bacteria species in 8 of the 18 positive samples were identical to those from the nasal swab culture results from the same nostril.

Conclusion: In ordinary otolaryngology practices, there are significant risks for bacterial contamination from the nasal vestibule to the tip of the Venturi atomizer even without direct contact. Clinicians must be more aware of this pattern of contamination, which has not been reported in the existing literature.

Key words: Venturi atomizer, bacteria, contaminations, aerosols, airflow, nasal, spray, patient safety, sterilization, compressed air

\section{Introduction}

In common otolaryngology practices, Venturi-principle atomizers are often used to apply topical anesthetics, vasoconstrictors, or other topical medications to the mucous membranes of the nose, throat, and oral cavity ${ }^{(1-4)}$. The principles of this atomizer rely on compressed air to aerosolize medications. It possess the advantage of having a large capacity reservoir for the desired medication and is able to hold multiple applications before the medication must be replaced. Another major advantage of the device is due to the use of compressed air. This enables the driving force of the delivery to be generally higher compared with hand-driven atomizers and is thus ideal for carrying the medication into narrowed or deep channels and cavities that are faced in common otolaryngology practices.

The major concern for the use for Venturi-principle atomizers 
has been the risk of contamination. The device was designed to be multiple-use, and many of the components of the device are made of plastic and rubber, preventing it from being autoclaved. Some studies have claimed that there is no risk of cross-contamination of patients with the use of the Venturi system atomizer ${ }^{(5,6)}$. However, many studies have revealed that after a period of use, bacteria tend to colonize many parts of the Venturi device ${ }^{(7-}$ ${ }^{10)}$, which has raised the issue of cross-contamination. Southwick et al. even reported that a contaminated atomizer may have caused a cluster of tuberculosis infections in North Carolina (11). Therefore, some researchers have proposed the use of singleuse or specially designed positive displacement atomizer. The use of such atomizers has greatly reduced the bacterial colonization on these devices ${ }^{(9)}$. Nevertheless, we have observed a new route of bacterial contamination during the use of Venturi atomizers. We noticed that when the medication was sprayed into a narrowed space, such as the nasal cavity, the jet of the compressed air was very strong and tended to rebound while it reached the narrowed cavity. This rebound airflow, combined with the aerosol, easily contacted the tip of the atomizer, potentially contaminating the device. The purpose of this study is to evaluate the possibility of the reverse jet flow created by using the Venturi atomizer to carry bacteria from the nasal cavity, thereby contaminating the atomizer tip.

\section{Materials and methods}

Atomizer nozzle tip cap

A specially designed cap to be attached to the atomizer tip was made by removing the anterior half of a pipette tip, as shown in Figure 1. This shortened tip can be attached over the tip of the atomizer nozzle, and the opening of the anterior end remained large enough without interrupting the spray jet while the atomizer was used. All caps were autoclave-sterilized before use.

\section{Reverse jet contamination}

Fifteen healthy subjects were enrolled in the study, and each nostril was regarded as a separate sample. A total of 30 samples were tested and collected. Before the test began, the Venturi atomizer (Nagashima Medical Instruments, Tokyo, Japan) was sterilized by immersion in $70 \%$ alcohol for 10 minutes before each use. The reservoir was filled with sterile saline. The sterile nozzle caps were attached before each spray and then collected immediately after each spray. The caps along with the collected aerosol particles were placed in a test tube for further bacterial culture. The first spray was aimed at the lumen of a sterile $5 \mathrm{ml}$ syringe, and the results of the cap cultures were regarded as negative controls. Following the first spray, a new sterile nozzle cap was used, and the following 3 sprays were aimed at one side of the nasal cavity of an enrolled test subject. After the spray, the cap along with the collected aerosols were immediately placed into the culture medium and sent for culture.

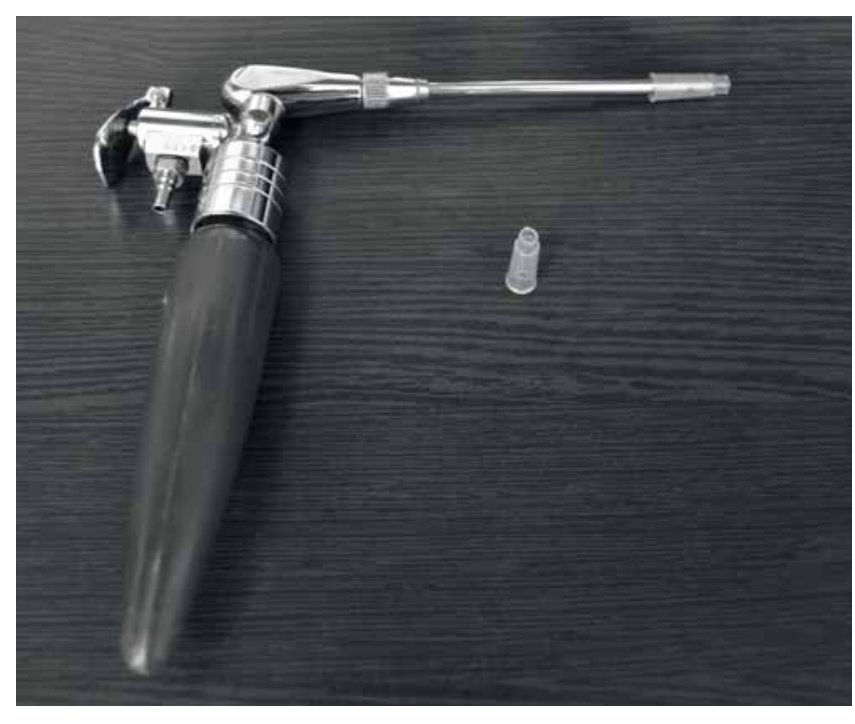

Figure 1. The Venturi atomizer and the specially made nozzle tip cap. The specially designed cap to be attached to the nozzle of the Venturi atomizer was made by removing the anterior half of a pipette tip.

Nasal vestibule swab culture Immediately following the spray experiment, nasal vestibule swab cultures were collected from the test subject as bacterial references for the previous experiments.

\section{Droplet depositions carried by the reverse jet airflow} In the demonstration of droplet deposition pattern resulted from the reverse jet airflow, a $3 \mathrm{ml}$ syringe lumen was used as a target representing the nasal cavity. A cotton ball soaked with black ink was placed in the syringe lumen. The simulated target was then struck by the compressed air using the atomizer at a distance of $1 \mathrm{~cm}$ for 0.5 seconds. The depositions of the generated ink droplets under atomizer air pressure settings of $0.3,0.6$, and $0.9 \mathrm{Mpa}$ were recorded with grid papers.

\section{Results}

As shown in Table 1, all 30 samples from the control group were negative for bacterial growth, and all of the samples from the nasal vestibule swab culture group were positive for bacterial growth. In the nasal swab group, Staphylococcus epidermidis and Staphylococcus aureus were most commonly found (11/30 and $6 / 30$, respectively). Other species, such as those from the Klebsiella, Corynebacterium, Enterobacter, and Citrobacter families, were also noted.

\section{Reverse jet contamination}

In the reverse jet tip culture group, 18 out of 30 samples (60\%) were positive for bacterial growth, confirming the bacterial contamination from the nasal vestibule to the atomizer tip through the reverse jet airflow. All of the microorganisms in this group 


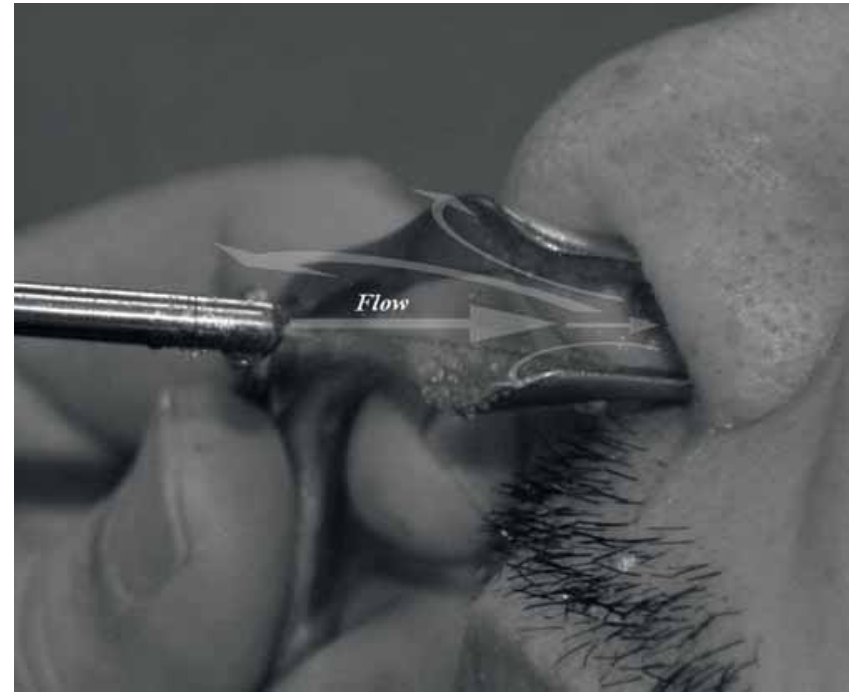

Figure 2. Venturi atomizer nasal applications. During the spray process, remarkable aerosols were noted traveling backwards through the reversed jet flow and attaching to the nozzle tip.

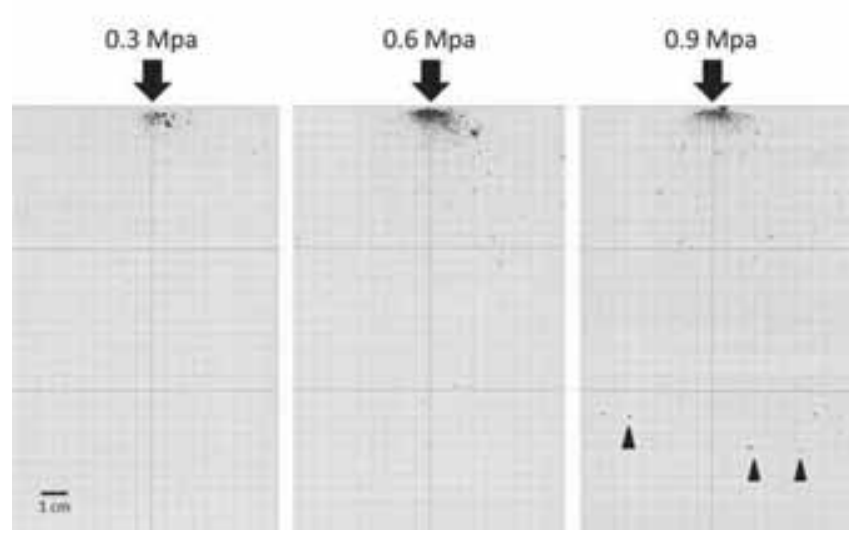

Figure 3. Droplet depositions carried by the reverse jet airflow. The deposition of ink droplet under the atomizer air pressure settings of $0.3,0.6$, and $0.9 \mathrm{Mpa}$. When the pressure was set to $0.3 \mathrm{Mpa}$, most of the aerosol depositions were found to be within the $10 \mathrm{~cm}$ range from the nasal cavity target. Arrow: position of the simulated target and direction of the reverse jet airflow. Arrow head: representative ink droplets. belong to the Staphylococcus family. The bacteria species in 8 of the 18 positive samples were identical to those in the nasal swab culture results from the same nostril.

\section{Droplet depositions carried by the reverse jet airflow}

As shown in Figure 3, under the atomizer air pressure settings of $0.9 \mathrm{Mpa}$, a significant number of the ink droplets carried by the reverse jet airflow were deposited more than $10 \mathrm{~cm}$ away from the struck nasal cavity target (arrow head). The amount of these depositions decreased when the pressure declined to 0.6 $\mathrm{Mpa}$. When the pressure was set to $0.3 \mathrm{Mpa}$, most of the droplet depositions were found to be within the $10 \mathrm{~cm}$ range from the target.

\section{Discussion}

The results of our study showed a new route of bacterial contamination that has not been reported in the existing literature. We have demonstrated that the use of a Venturi atomizer towards a narrowed space, such as the nasal cavity, can possibly dislodge bacteria in the narrow cavity by the force of the spray, with the bacteria carried backward to contaminate the nozzle tip. Moreover, the particles carried by the reverse airflow were able to contaminate a fan-shaped area with its size proportional to the compressed air pressure of the atomizer.

Before performing examinations, such as a transnasal fiberscopy, or procedures, such as foreign body removal, in the practice of otolaryngology, topical anesthetics and vasoconstrictive agents are usually sprayed into the nares or the throat to improve patient comfort. In many institutions, especially in Asian countries, this treatment is accomplished with a positive-pressure Venturi system atomizer.

The Venturi system atomizer is based on pressure-volume-flow dynamics: compressed air is forced into/across a liquid reservoir that houses an outlet tube that allows for the expression of a mist of anesthetic and/or vasoconstrictive agents. The standard method of administering solutions into the nasal fossae has been through the use of one of a variety of atomizers, which incorporates a hand-held bellows and works on the Venturi principle. The nozzle of an atomizer consists of a fine-bore inner tube contained within a larger outer tube, which is connected to the hand-held bellows. The inner tube is placed into the liquid within the reservoir of the atomizer, which is drawn up and expelled from the tip of the nozzle as a fine spray on compression of the bellows.

Since the development of the Venturi atomizer, many researchers have noticed potential contamination problems while using this device. Most of the studies have focused on the bacterial colonization on the device during a period of use. The concerns appeared to be obviously logical as the Venturi atomizer device is not designed to be disposable after a single use. Coakley et al. first raised this concern in 1993 and stated that the Venturi nasal sprays have the disadvantage of suck-back, which makes them unhygienic for use in more than one patient (12). The authors even claimed to have developed a disposable nasal spray system, which, however, did not become universally popular. As mentioned by Sraggs et al., "the devices are rarely cleaned, nor is the cocaine in the reservoir changed between patients". Although there have been some reports suggesting 
Table 1. Bacterial culture data from the control, reverse jet, and nasal swab culture groups.

\begin{tabular}{|c|c|c|c|}
\hline Case Number & Control & Spray nozzle & Vestibular tip culture \\
\hline 1 & no growth & Staphylococcus epidermidis & Staphylococcus aureus \\
\hline 2 & no growth & no growth & Klebsiella pneumonia \\
\hline 3 & no growth & no growth & Corynebacterium urealyticum \\
\hline 4 & no growth & Staphylococcus epidermidis & Staphylococcus hominis \\
\hline 5 & no growth & Staphylococcus paseruri; hominis & Klebsiella pneumonia; Staphylococcus capitis/epidermidis \\
\hline 6 & no growth & Staphylococcus aureus & Staphylococcus aureus \\
\hline 7 & no growth & Staphylococcus aureus & Staphylococcus aureus \\
\hline 8 & no growth & Staphylococcus epidermidis & Klebsiella pneumonia; Staphylococcus capitis/epidermidis \\
\hline 9 & no growth & no growth & Klebsiella pneumoniae; Staphylococcus aureus \\
\hline 10 & no growth & Staphylococcus capitis/hominis & Enterobacter aerogenes/Staphylococcus epidermidis \\
\hline 11 & no growth & no growth & Citrobaceter freundii \\
\hline 12 & no growth & Staphylococcus schleiferi ssp coagulans & Escherichia coli; Staphylococcus epidermidis \\
\hline 13 & no growth & Staphylococcus aureus & Klebsiella pneumonia; Staphylococcus aureus \\
\hline 14 & no growth & Staphylococcus epidermidis & Staphylococcus epidermidis \\
\hline 15 & no growth & Staphylococcus epidermidis & Enterobacter aerogenes; Staphylococcus hyicus \\
\hline 16 & no growth & Staphylococcus epidermidis & Klebsiella oxytoca; Staphylococcus aureus \\
\hline 17 & no growth & no growth & Enterobacter cloacae; Staphylococcus aureus \\
\hline 18 & no growth & Staphylococcus epidermidis & Enterobacter aerogenes; Staphylococcus epidermidis \\
\hline 19 & no growth & Staphylococcus epidermidis & Enterobacter aerogenes; Staphylococcus saprophyticus \\
\hline 20 & no growth & no growth & Staphylococcus epidermidis \\
\hline 21 & no growth & no growth & Staphylococcus capitis \\
\hline 22 & no growth & no growth & Staphylococcus lugdunensis \\
\hline 23 & no growth & no growth & Klebsiella pneumonia; Staphyloccus epidermidis \\
\hline 24 & no growth & no growth & Staphylococcus aureus \\
\hline 25 & no growth & Staphylococcus epidermidis & Staphylococcus hominis \\
\hline 26 & no growth & Staphylococcus epidermidis & Enterobacter gergoviae; staphylococcus simulans \\
\hline 27 & no growth & no growth & Enterobacter gergoviae; staphylococcus simulans \\
\hline 28 & no growth & Staphylococcus epidermidis & Staphylococcus epidermidis \\
\hline 29 & no growth & Staphylococcus epidermidis & Staphylococcus epidermidis \\
\hline 30 & no growth & no growth & Klebsiella pneumoniae; Staphylococcus epidermidis \\
\hline
\end{tabular}

that Venturi-type atomizers may not have a high potential contamination risk and possibly do not pose an infectious risk of bacterial transmission between patients, the risk of contaminations cannot be ignored ${ }^{(5,6,13)}$.

New devices, such as positive displacement atomizers, have been developed and proved to be superior to Venturi atomizers in reducing bacterial contamination ${ }^{(9)}$. These devices shared the common features of adopting a closed reservoir design and a hand-driven fluid dispersal mechanism. Although these designs stay away from the use of external compressed air and minimize the "suck-back" phenomenon associated with a Venturi atomizer, 
the force needed to disperse and carry the desired medication into deep cavities is greatly reduced. Moreover, when the handdriven compressing mechanism is used on an atomizer, keeping the nozzle precisely aimed towards a small cavity while trying to compress the atomizer bottle by hand at the same time is difficult.

If a compressed air-driven force is to be used to deliver the medication for the desired distance, the bacterial aerosols resulting from the relatively strong airflow must be taken into consideration. During the spray process, as shown in Figure 2, remarkable aerosols were noted traveling backwards through the reversed jet flow and attaching to the nozzle tip. Bacterial air contamination has often been discussed in the field of dentistry as this problem increases during dental treatments. Klyn et al. recommended the use of a suction-type aerosol reduction device to significantly reduce the bacterial contamination during ultrasonic scaling ${ }^{(14)}$. Nevertheless, little attention has been paid to this issue in the field of otolaryngology.

For the first time, we have shown that even without direct contact, bacteria can translocate from the nares to the tip of the Venturi atomizer. We believe that this contamination results from the air contamination generated by the compressed air jet flow striking the surface of the nares. In a recent study conducted in Japan, the researchers concluded that the detected bacteria contaminating the atomizer had originally colonized the nares ${ }^{(7)}$. Their results also implied that the route of this contamination was directly from the nose to the atomizer tip, which are compatible with the findings of our study. One of the limitations of our study is that we did not prove that the bacteria attached to the nozzle tip were able to be further transmitted by the following sprays or to spread backwards into the device or the reservoir. Although low numbers of bacteria do not necessarily result in clinical significance, the potential threats of this contamination were noteworthy especially with hazardous pathogens such as Mycobacterium tuberculosis or certain viruses. It is known that many pathogens can be transmitted through aerosols ${ }^{(15-17)}$. There is already evidence that $M$. tuberculosis can be transmitted through the nasal-only route ${ }^{(18,19)}$. The aerosol transmission of influenza $A$ virus has also been well addressed ${ }^{(20)}$. Furthermore, the results in our study also raised a serious concern that during the use of the Venturi atomizer, we were in fact creating an outspread flow of droplets and aerosols containing particles from the nares of the patient, which may expose the patient as well as the health-care workers to potential threats.

For the device sterilization, some researchers have suggested that wiping the device with isopropyl alcohol can eliminate microbial growth for a 2-week interval ${ }^{(8)}$. Aydin et al. proposed additional precautions, such as the use of protective punched caps or rinsing the bottle tip with alcohol ${ }^{(21)}$. As to the airborne infection control, Simonds et al. suggested that during dropletaerosol producing procedures, health-care workers working within $1 \mathrm{~m}$ distance of an infected patient should have a higher level of respiratory protection ${ }^{(22)}$. Respiratory and facial protections were also highly recommended ${ }^{(23)}$. According to our current findings, sterilization of the outside surface of the atomizer tip might not be sufficient for patient safety. A re-design of the Venturi atomizer with disposable nozzles along with aerosol reduction devices was encouraged, and before the new device becomes available, we recommend the device sterilization procedure to be performed throughout the outer surface of the atomizer and even thoroughly immersing the entire atomizer tip into $70 \%$ alcohol to sterilize the inner chamber of the tip after each use. The pressure of the compressed air delivered by the atomizer was also recommended to be maintained at a minimum applicable level to reduce the generated droplets and aerosols.

\section{Conclusion}

In ordinary otolaryngology practice there are significant risks for bacterial contaminations from the nasal vestibule to the tip of a Venturi atomizer even without direct contact. The contamination may be related to the reversed jet airflow created during the atomizer use. Clinicians must be more aware of this pattern of contamination, which has not been reported in the existing literature.

\section{Authorship contribution}

TMT: Drafting the manuscript, acquisition of data; PYC: Analysis of data; $\mathrm{HT}$ : Revising the manuscript; $\mathrm{HCL}$ : Revising the manuscript; CYC: Revising the manuscript; $\mathrm{SHH}$ : Conception and design of study, analysis of data, revising the manuscript.

\section{Acknowlegdement}

None

\section{Conflicts of Interest}

The authors declare no conflicts of interest.

\section{References}

1. Denlinger JK, Ellison N, Ominsky AJ Effects of intratracheal lidocaine on circulatory responses to tracheal intubation.
Anesthesiology. 1974; 41: 409-412.

2. Schonemann NK, van der Burght M, ArendtNielsen L, Bjerring P. Onset and duration of hypoalgesia of lidocaine spray applied to oral mucosa--a dose response study. Acta Anaesthesiol Scand. 1992; 36: 733-735.

3. Matthys $\mathrm{H}$, Knoch M, Eltschka R. A new aerosol device for bronchial provocation tests. 
Respiration 1993: 60:343-350.

4. Noorily AD, Otto RA, Noorily SH. Intranasal anesthetic effects of lidocaine and tetracaine compared. Otolaryngol Head Neck Surg. 1995; 113: 370-374.

5. Scianna JM, Chow JM, Hotaling A. Analysis of possible cross-contamination with the Venturi system atomizer. Am J Rhinol 2005; 19: 503-507.

6. Visosky AM, Murr AH, Ng V, Dentoni T, Weir L, Haller BL. Multiple-use atomizers in outpatient otolaryngology clinics are not necessarily an infectious risk. Otolaryngol Head Neck Surg. 2003; 128: 447-451.

7. Ikeda K, Sakai Y, Haruyama T, et al. Bacterial contamination of multiple-use atomizers commonly used in Japan. Indian J Otolaryngol Head Neck Surg. 2009; 61: 193196.

8. Dubin MG, White DR, Melroy CT, Gergan MT, Rutala WA, Senior BA. Multi-use Venturi nasal atomizer contamination in a clinical rhinologic practice. Am J Rhinol. 2004; 18: 151-156.

9. Wolfe TR, Hillman TA, Bossart PJ. The comparative risks of bacterial contamination between a venturi atomizer and a positive displacement atomizer. Am J Rhinol. 2002; 16: 181-186.

10. Spraggs PD, Hanekom WH, Mochloulis G, Joseph T, Kelsey MC. The assessment of the risk of cross-infection with a multi-use nasal atomizer. J Hosp Infect. 1994; 28: 315-321.

11. Southwick KL, Hoffmann K, Ferree K, Matthews J, Salfinger M. Cluster of tuberculosis cases in North Carolina: possible association with atomizer reuse. Am J Infect Control. 2001; 29: 1-6.

12. Coakley JF, Arthurs GJ, Wilsher TK. The need for and development of a single use disposable nasal spray. J Laryngol Otol. 1993; 107: 20-23.

13. Rizzi M, Batra PS, Hall G, Citardi MJ, Lanza DC. An assessment for the presence of bac terial contamination of Venturi principle atomizers in a clinical setting. Am J Rhinol. 2005; 19: 21-23.

14. Klyn SL, Cummings DE, Richardson BW Davis RD. Reduction of bacteria-containing spray produced during ultrasonic scaling. Gen Dent 2001; 49:648-652.

15. Cole EC, Cook CE. Characterization of infectious aerosols in health care facilities: an aid to effective engineering controls and preventive strategies. Am J Infect Control. 1998; 26: 453-464.

16. Wang B, Zhang A, Sun JL, Liu H, Hu J, Xu LX. Study of SARS transmission via liquid droplets in air. J Biomech Eng. 2005; 127: 32-38

17. Tang JW, Li Y, Eames I, Chan PK, Ridgway GL. Factors involved in the aerosol transmission of infection and control of ventilation in healthcare premises. J Hosp Infect. 2006; 64: 100-114.

18. Roy CJ, Milton DK. Airborne transmission of communicable infection--the elusive pathway. N Engl J Med. 2004; 350: 1710-1712.

19. Louveau C, Descroix D, Garnier L, et al. A nose-only apparatus for airborne delivery of Mycobacterium tuberculosis to mice: calibration of biological parameters. Microbes Infect. 2005; 7: 457-466.
20. Tellier R. Aerosol transmission of influenza A virus: a review of new studies. J R Soc Interface. 2009; 6 Suppl 6: S783-790.

21. Aydin E, Hizal E, Akkuzu B, Azap O. Risk of contamination of nasal sprays in otolaryngologic practice. BMC Ear Nose Throat Disord. 2007; 7: 2

22. Simonds $A K$, Hanak $A$, Chatwin $M$, et al. Evaluation of droplet dispersion during non-invasive ventilation, oxygen therapy, nebuliser treatment and chest physiotherapy in clinical practice: implications for management of pandemic influenza and other airborne infections. Health Technol Assess. 2010; 14: 131-172.

23. Bunyan D, Ritchie L, Jenkins D, Coia JE Respiratory and facial protection: a critical review of recent literature. J Hosp Infect. 2013; 85: 165-169.

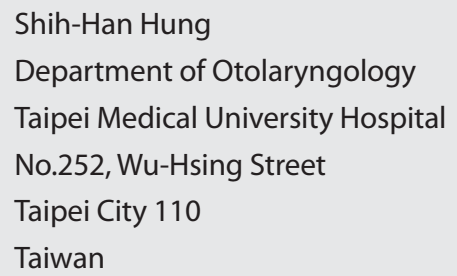

Shih-Han Hung

Department of Otolaryngology Taipei Medical University Hospital No.252, Wu-Hsing Street Taipei City 110

Taiwan

Tel: $+886-2-2737-2181$

Fax: +886-2-2836-9275

E-mail: seedturtle@gmail.com 